\title{
Effect of zero field splitting interactions on the paramagnetic relaxation enhancement of nuclear spin relaxation rates in solution
}

\author{
Robert R. Sharp \\ Department of Chemistry, The University of Michigan, Ann Arbor, Michigan 48109
}

\begin{abstract}
(Received 25 August 1992; accepted 5 October 1992)
\end{abstract}
\begin{abstract}
The enhancement of nuclear spin relaxation rate $R_{1 m}$ that is produced by paramagnetic metal ions in solution (the NMR-PRE) has been investigated for electron spin systems with $S=1$ using recently developed relaxation theory that incorporates both Zeeman and zero field splitting (zfs) interactions of arbitrary magnitude in the electron spin Hamiltonian. The zfs interaction gives rise to important qualitative features which have no analog in the Zeeman-limit theory. The three principal physical phenomena responsible for these effects are (1) alterations in the geometry of the magnetic dipole-dipole coupling energy due to requantization of the electron spin from laboratory to molecular axes; (2) the crossing or "pinching" of spin energy levels that occurs in the regime of field strengths between the zfs and Zeeman limits; and (3) an effective magnetic field dependence in the reorientational correlation time that results from a change in the appropriate definition of this quantity in the intermediate regime. In the zfs limit and in the intermediate regime, the field dispersion profile depends strongly on the position of the nuclear spin with respect to the molecular coordinate axes. For equatorial positions of the nuclear spin, the principle qualitative feature of the dispersion profile is a strong increase in $R_{1 m}$ with increasing field strength coupled, in most cases, with a shallow local $R_{1 m}$ maximum; both features are centered near the cross-over field between the limits. For axial positions, the profile exhibits a feature that is superficially similar to those characteristic of Zeeman-limit theory, but which is fundamentally different in quantitative properties and in physical origin. As a test of theoretical predictions, the experimental magnetic field profile of the NMR-PRE of the hexaquo-Ni(II) cation, an $S=1$ model system that has previously been studied extensively, has been reinterpreted. It is shown that the major qualitative features of the experimental field profile result specifically from physical effects of the zfs interaction and are closely related to the phenomenon of requantization of the electron spin in the intermediate regime.
\end{abstract}

\section{INTRODUCTION}

Small concentrations of paramagnetic metal ions in solution frequently produce profound enhancements of the spin relaxation rates $\left(R_{1 m}, R_{2 m}\right)$ of nuclei of ligands and coordinated solvent molecules, a phenomenon referred to as the nuclear magnetic resonance (NMR) paramagnetic relaxation enhancement, or NMR-PRE. The theory of the NMR-PRE dates from the late 1950 s and has since been used widely to investigate the chemical and magnetic environments of paramagnetic metal ions in chemical and biological systems. More recently, the NMR-PRE has assumed additional practical importance with respect to NMR imaging applications in medicine.

The classical theory of the NMR-PRE was developed by Solomon, ${ }^{1}$ Bloembergen, ${ }^{2,3}$ and Morgan ${ }^{3}$ (SBM theory). SBM theory is a Zeeman-limit theory in that it assumes that the Zeeman interaction comprises the largest portion of the static electron spin Hamiltonian $H_{S}$, i.e.,

$$
\hbar^{-1} H_{S} \equiv \mathscr{H}_{S}=\mathscr{H}_{Z}+\mathscr{H}_{\mathrm{zfs}}+\mathscr{H}_{\mathrm{hf}} \quad\left(\mathscr{H}_{Z} \gg \mathscr{H}_{\mathrm{zfs}}, \mathscr{H}_{\mathrm{hf}}\right) \text {, }
$$

where terms on the right-hand side represent, respectively, the Zeeman, zero field splitting (due to electronic quadrupole couplings and electron-electron magnetic dipole couplings), and nuclear hyperfine portions of the spin Hamil- tonian. In the Zeeman limit, the precessional motion of the clcctron spin is quantized by the external magnetic field. In contrast, when the zfs energy is larger than the Zeeman energy $\left(\mathscr{H}_{\text {zfs }} \gg \mathscr{H}_{Z}\right)$, the quantization axis of the electronic precessional motion changes to a molecule-fixed coordinate system that is aligned along the principal axes of the zfs tensor. This change in quantization axis has profound effects on the functional form of the NMR-PRE, as is described in some detail below. Zeeman-limit theory is generally applicable to solutions containing $S=1 / 2$ radicals, as well as to ions with orbital singlet ground states, especially when the spin multiplicity of the ground state prevents first-order mixing with low-lying excited states, as for $\mathrm{Mn}$ (II) and Gd(III). Other ions with $S \geqslant 1$ usually experience much larger zero field splitting interactions, and for the relaxation properties of these ions, the $\mathrm{zfs}$ interaction frequently has important consequences.

In previous work, I have developed a theory which parallels the form of SBM theory, but is appropriate to the zfs limit. ${ }^{4,5}$ Subsequently, a more general theory was developed $^{6}$ which bridges the two limiting regimes, subject to an assumption of "slow motion" conditions in the intermediate regime, i.e., that electron spin relaxation is faster than molecular tumbling $\tau_{S}<\tau_{R}$. (This assumption is not required by either of the limiting theories.) The present study explores systematically the influence of the zfs inter- 
action on the NMR-PRE phenomenon for $S=1$ systems. It is shown that several previously unrecognized physical phenomena which originate specifically in the zfs interaction contribute to the magnetic field dependence of NMRPRE data (i.e., to the shape of the "magnetic field dispersion profile" of the PRE). Of particular significance are (1) the geometric effects of the requantization of the electron spin; (2) the crossing or "pinching" of spin encrgy levels that occurs in the regime of field strengths between the zfs and Zeeman limits; and (3) effective magnetic field dependence in the definition of the reorientational correlation time in the intermediate regime. These three factors produce new, and previously unrecognized qualitative features in the field dispersion profile for spin systems that are outside the Zeeman limit. The physical origins of these effects and their influence on the functional form of the profile are described below and illustrated through theoretical calculations.

An alternative theoretical approach to that used herc has been developed ${ }^{7-9}$ based on the stochastic Liouville equation of motion. In essence, the combined Liouville superoperator of the spin and lattice variables is constructed and then diagonalized to yield the time correlation function $\langle S(t) \cdot S(0)\rangle$ needed for calculation of the nuclear spin $T_{1}$. My theoretical approach, which is more closely related to that of Lindner ${ }^{10}$ and Friedman et al., ${ }^{11}$ describes each portion of the dipolar coupling Hamiltonian in its natural coordinate system, i.e., zfs interactions are described in the molecular coordinate system and Zeeman interactions in the laboratory coordinate system, and then relates these coordinate systems to the laboratory frame using the known transformation properties of spherical tensors. A very important advantage of this approach is its retention in explicit algebraic form of those parts of the dipolar interaction which originate in the $\mathrm{zfs}$ splittings. This clarifies various geometrical and physical aspects of the problem, especially those relating to the $\mathrm{zfs}$, which are not readily apparent when viewed from the laboratory system alonc.

The NMR-PRE of aqueous Ni(II) solutions has been studied intensively both experimentally and theoretically ${ }^{11-18}$ as a model $S=1$ spin system in which the zfs is important $\left(\mathscr{H}_{\mathrm{zfs}}>\mathscr{H}_{Z}\right)$ over much of the accessible range of magnetic field strengths. The experimental data, specifically the magnetic field profile of the NMR-PRE, exhibits a curious functional form in which the relaxation rate $R_{1 m}$ of protons in the inner coordination sphere of the metal ion undergoes a small gradual rise in the low field regime (0.04-0.6 T), then falls rapidly, passing through a local minimum in the neighborhood of $4 \mathrm{~T}$ and rising again at the higher field strengths. The major qualitative feature of this profile, viz. the local $R_{1 m}$ minimum near $4 \mathrm{~T}$ (but not the rise at low field) can be simulated with acceptable rms error by Zeeman-limit theory, but this fit leads to physically unlikely values of the theoretical parameters and is probably fortuitous from a physical standpoint (see below). The inadequacy of Zeeman-limit theory has been recognized in previous work, ${ }^{11,16-18}$ but the profound influence of the zfs on the qualitative form of the field disper- sion profile has not. It is shown here that the principal physical phenomena that determine the profile's shape originate in the $\mathrm{zfs}$ interaction and the effect of the $\mathrm{zfs}$ on the quantization of the $S$ spin. These phenomena, which are ennumerated briefly above, have no parallel in Zeeman limit theory and have not been described previously in the literature. Their inclusion is essential to theoretical interpretations of NMR-PRE phenomena of spin systems that are outside the Zeeman limit.

\section{METHODS}

The paramagnetic relaxation enhancement of a nuclear spin in the coordination complex of a paramagnetic solute, $R_{1 m}$, results from time dependence in the dipolar and scalar parts of the electron-nuclear hyperfine energy: $R_{1 m}$ $=R_{1 m, \text { dip }}+R_{1 m, \text { sc }}$. From zfs-limit theory, ${ }^{4,6}$

$$
\begin{aligned}
R_{1 m, \mathrm{dip}}^{(\mathrm{zfs})}= & \frac{4}{3}\left(\frac{\gamma_{1} g \beta_{0}}{r^{3}}\right)^{2}\left(\frac{\mu_{0}}{4 \pi}\right)^{2}\left\{\frac{S(S+1)}{3}\right. \\
& \times\left[1+P_{2}(\cos \theta)\right] j\left(\omega_{I}\right)+(2 S+1)^{-1} \\
& \left.\times\left[1-2^{-1} P_{2}(\cos \theta)\right] \sum_{\mu}\left|c_{S, \mu}^{+}\right|^{2} j\left(\omega_{\mu}\right)\right\} .
\end{aligned}
$$

$\gamma_{I}$ is the magnetogyric ratio of the $I$ spin, $\omega_{I}=\gamma_{I} B$ is the nuclear Larmor frequency, $g$ is the electronic $g$ factor, $\beta_{0}$ is the Bohr magneton, $\mu_{0}$ is the magnetic permeability of free space, $r$ is the $I-S$ distance, $\Theta$ is the polar angle of the $I-S$ vector in the molecular coordinate frame, and $P_{2}(\cos \theta)$ is the second-order Legendre polynomial in $\cos \Theta . j(\omega)$ $=\tau_{c} /\left(1+\omega^{2} \tau_{c}^{2}\right)$ is the spectral density function and $\tau_{c}$ is the correlation time of the magnetic dipole interaction. The sum is over the basis states $|\mu\rangle$ of $\mathscr{H}_{\text {zfs, }}$ and $c_{S, \mu}^{+}$is the raising operator of the $S$ spin manifold. For $S=1$, two transitions contribute to the sum, one with $\mu=-1, \omega_{\mu}$ $=\omega_{D},\left|c_{1,-1}^{+}\right|^{2}=1$, the other with $\mu=0, \omega_{\mu}=\omega_{D},\left|c_{1,0}^{+}\right|^{2}$ $=1$, where $\omega_{D}=2 \pi c D$ is the uniaxial zfs splitting parameter $D\left(\mathrm{~cm}^{-1}\right)$ expressed in $\mathrm{rad} \mathrm{s}^{-1}$. With these substitutions, Eq. (2) reduces, for $S=1$, to

$$
\begin{aligned}
R_{\mathrm{I} m, \mathrm{dip}}^{\mathrm{z} f \mathrm{~s})}= & \frac{8}{9}\left(\frac{\gamma_{I} \mathrm{~g} \beta_{0}}{r^{3}}\right)^{2}\left(\frac{\mu_{0}}{4 \pi}\right)^{2}\left\{\left[1+P_{2}(\cos \Theta)\right] j\left(\omega_{I}\right)\right. \\
& \left.+\left[1-2^{-1} P_{2}(\cos \Theta)\right] j\left(\omega_{D}\right)\right\} .
\end{aligned}
$$

The Zeeman-limit expression corresponding to Eq. (2) is ${ }^{2}$

$$
\begin{aligned}
R_{1 m, \mathrm{dip}}^{(Z)}= & \left(\frac{2}{15}\right)\left(\frac{\mu_{0}}{4 \pi}\right)^{2}\left(\frac{\gamma_{I} \mathrm{~g} \beta_{0}}{r^{3}}\right)^{2} S(S+1)\left[3 j_{1}\left(\omega_{1}\right)\right. \\
& \left.+j_{2}\left(\omega_{S}-\omega_{I}\right)+6 j_{2}\left(\omega_{S}+\omega_{I}\right)\right]
\end{aligned}
$$

where $\omega_{S}$ and $\omega_{I}$ are the electronic and nuclear Larmor frequencies.

The definition of the dipolar correlation time differs in the $\mathrm{zfs}$ and Zeeman limits. In the former,

$$
\left(\tau_{c}\right)_{\mathrm{zfs}}^{-1}=\left(\tau_{S_{2}}\right)^{-1}+\left(\tau_{R}^{(1)}\right)^{-1}
$$

and in the latter,

$$
\left(\tau_{c_{1,2}}\right)^{-1}=\left(\tau_{S_{1,2}}\right)^{-1}+\left(\tau_{R}^{(2)}\right)^{-1},
$$


where $\tau_{R}^{(1,2)}$ are reorientational correlation times for first and second rank molecule-fixed tensors. According to classical diffusion theory, $\tau_{R}^{(1)}=3 \tau_{R}^{(2)}$ (Ref. 19). $\tau_{S_{1}}$ and $\tau_{S_{2}}$ are the electron spin relaxation times parallel to, and in the plane transverse to, the axis of the electronic precessional motion. In previous work, ${ }^{4-6}$ a single parameter $\tau_{S}$ was used. Usually the available information about the physical processes of electron spin rclaxation is not sufficiently detailed to justify more elaborate treatments. However, it should be noted that the definition of $\tau_{S}$ differs in the zfs and Zeeman limits; the possible effect of this is discussed below.

For scalar $T_{1}$ relaxation, the zfs- and Zeeman-limit expressions are, respectively, ${ }^{2,4}$

$$
\begin{aligned}
R_{1 m, \mathrm{sc}}^{(\mathrm{zfs})}= & \frac{A^{2}}{3}\left\{\frac{2}{3} S(S+1) j_{S}\left(\omega_{I}\right)+(2 S+1)^{-1}\right. \\
& \left.\times \sum_{\mu}\left|c_{S, \mu}^{+}\right|^{2}\left[j_{S}\left(\omega_{\mu}-\omega_{I}\right)+j_{S}\left(\omega_{\mu}+\omega_{I}\right)\right]\right\}
\end{aligned}
$$

and

$$
R_{1 m, \mathrm{sc}}^{(Z)}=\frac{2}{3} A^{2} S(S+1) j_{S}\left(\omega_{S}-\omega_{1}\right) .
$$

$A$ is the scalar coupling constant in $\mathrm{rad} \mathrm{s}^{-1}$ and $j_{s}(\omega)$ $=\tau_{\mathrm{sc}} /\left(1+\omega^{2} \tau_{\mathrm{sc}}^{2}\right)$. As is true for the dipolar correlation time, the scalar correlation time $\tau_{\mathrm{sc}}$ differs in the zfs and Zeeman limits ${ }^{4}$

$$
\begin{aligned}
& \left(\tau_{\mathrm{sc}}\right)_{\mathrm{zfs}}^{-1}=\left(\tau_{S}\right)^{-1}+\left(\tau_{R}^{(1)}\right)^{-1}+\left(\tau_{\mathrm{ex}}\right)^{-1}, \\
& \left(\tau_{\mathrm{sc}}\right)_{\mathrm{z}}^{-1}=\left(\tau_{S}\right)^{-1}+\left(\tau_{\mathrm{ex}}\right)^{-1} .
\end{aligned}
$$

The zfs limit expression contains a term $\left(\tau_{R}^{(1)}\right)^{-1}$ due to the fact that the motion of the electron spin is quantized along molecule-fixed axes, and molecular reorientation leads to time dependence in the scalar Hamiltonian $\mathscr{H}_{\text {sc }}$ $=\mathbf{A} \overline{\mathbf{I}} \cdot \overline{\mathbf{S}} \cdot \tau_{\mathrm{ex}}$ is a correlation time for chemical exchange processes involving the $I$ spin (see below).

In the intermediate regime of field strengths ( $\mathscr{H}_{\mathrm{zfs}}$ $\left.\approx \mathscr{H}_{Z}\right), R_{1 m}$ was calculated using the computer program PARELAX, which is based on the theory of Ref. 6 .

When the resonant nuclear spin is not permanently bonded in the first coordination sphere of the paramagnetic complex, but rather undergoes chemical exchange reactions between "bound" sites in the complex and "free" sites in the diamagnetic solvent, the observed relaxation rate is an exchange-averaged quantity ${ }^{20}$

$$
R_{1 p} \equiv\left(T_{1 p}\right)^{-1}=\frac{f_{m}}{T_{1 m}+\tau_{\mathrm{ex}}}+\left(T_{1 p}\right)_{\mathrm{os}}^{-1}
$$

$f_{m}$ is the mole fraction, $\tau_{\mathrm{ex}}$ is the mean residence time, and $T_{1 m}$ is the nuclear spin relaxation time of $I$ spins in the bound site. Equation (10) assumes that there is only one chemically distinguishable class of bound sites and that $f_{m} \ll 1$. $\left(T_{1 p}\right)_{\text {os }}^{-1}$ is the outer sphere paramagnetic relaxation enhancement, for which the appropriate theoretical forms in the zfs and Zeeman limits are given in Refs. 5 and 21.

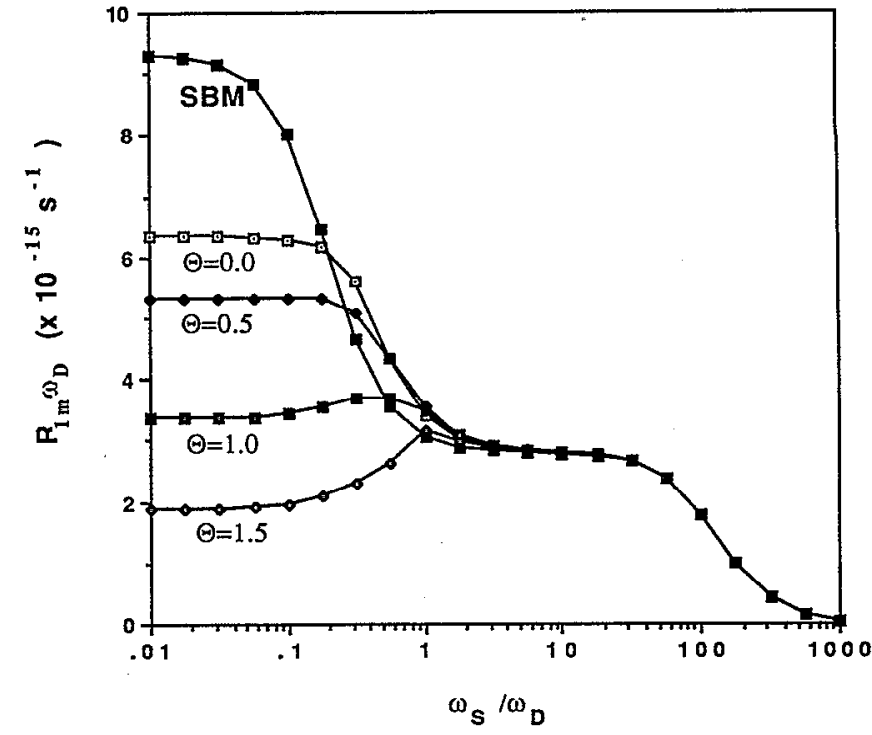

FIG. 1. Parametrized theoretical plots of $\omega_{D} R_{1 m}$ vs $\omega_{S} / \omega_{D}$ calculated for $S=1$ using the generalized theory of Ref. 6 , and for Zeeman limit theory (SBM) using Eqs. (3) and (5). Families of curves were calculated using the program PARELAX with the specified $\Theta$ values. Other theoretical parameters were $\omega_{D} \tau_{c}=5$ ( $\tau_{c}$ was taken to be field independent), $r_{I S}$ $=0.2657 \mathrm{pm}$, and $g=2$. The SBM result was calculated using the same parameters.

\section{RESULTS AND DISCUSSION}

Systematic studies of the form of the field dispersion profile of NMR-PRE for $S=1$ upon passage from the zfs to the Zeeman limit have been carried out using the theory of Ref. 6. A parametrized form of the theory was used in which angular frequencies and the nuclear spin relaxation rate $R_{1 m}$ are specified in units of $\omega_{D}$ (the uniaxial zfs splitting in rad s $\mathrm{s}^{-1}$ ) and correlation times in units of $\omega_{D}^{-1}$. The resulting field profiles are of $\omega_{D} R_{1 m}$ as a function of $\omega_{S} / \omega_{D}$, where the point $\omega_{S} / \omega_{D}=1$ corresponds to the midpoint between the Zeeman and zfs limits. The nuclear gyromagnetic ratio $\gamma_{I}$ and the interspin distance $r$ enter the theory only as a scaling factor $\left(\gamma_{I}^{2} / r^{6}\right)$, the value of which was taken to be $\left(r_{1}^{2} / r^{6}\right)=2.034 \times 10^{74}$ in S.I. units. This corresponds to protons at an $I-S$ distance of $0.2657 \mathrm{pm}$, which is appropriate to the aqueous $\mathrm{Ni}$ (II) system, which is considered below. However, the focus of this discussion is the effect of the zfs interaction on the qualitative shape of the field dispersion profile, and in this regard, scaling factors are irrelevant.

\section{A. Dependence on molecular geometry}

In the zfs limit, but not in the Zeeman limit, $R_{1 m}$ depends on the orientation of the $I-S$ vector in the molecular coordinate system which diagonalizes the $\mathrm{zfs}$ tensor. For a uniaxial zfs interaction (which is assumed here), $R_{1 m}$ depends on the polar angle $\Theta$ between the $I-S$ vector and the molecular $z$ axis. Figure 1 shows the variation in shape of the field dispersion profile for selected values of $\theta$ $(0 \leqslant \theta \leqslant \pi / 2)$. In these calculations, the dipolar correlation time $\omega_{D} \tau_{c}$ was set equal to 5 and was taken to be field 


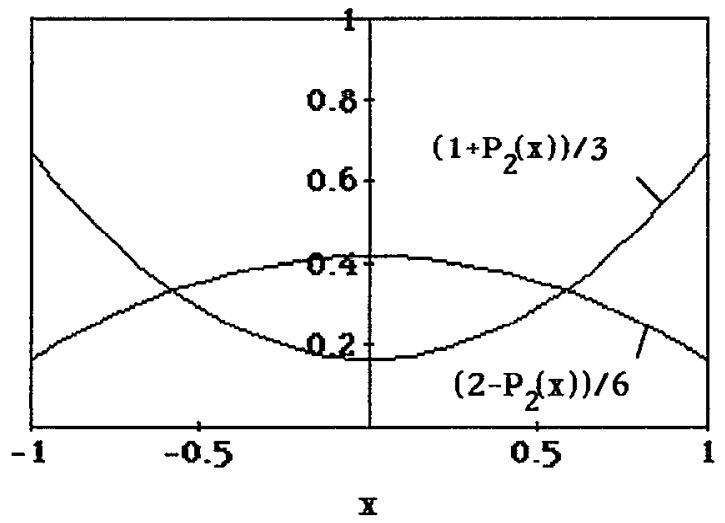

FIG. 2. Plots of the functions $\Phi_{0}=\left[1+P_{2}(x)\right] / 3$ and $\Phi_{1}=\left[2-P_{2}(x)\right] / 6$, which occur in the zfs-limit theory [Eqs. (2) and (2a)]. $P_{2}(x)$ is the second-order Legendre polynomial in the argument $x \equiv \cos \theta$, where $\Theta$ is the polar angle between the $I S$ vector and the unique axis of the molecular coordinate system which diagonalizes the zfs tensor.

independent. For comparison, the Zeeman limit (SBM) curve calculated using the same $\omega_{D} \tau_{c}$ is also shown in Fig. 1 (solid squares). When $\omega_{S} / \omega_{D} \gg 1$, the general theory coincides with SBM theory and the dependence on molecular geometry disappears.

The field dispersion profile in Zeeman limit theory (Fig. 1) exhibits the familiar form of two well-defined dispersions, one at lower field centered about $\omega_{s} \tau_{c}=1$, the other at higher field centered about $\omega_{I} \tau_{c}=1$, with relative amplitudes $7 / 3$ as given by Eq. (3).

The profiles for the zfs limit and the intermediate region are more complex. The zfs limit theoretical expression (2a) provides a basis for understanding the $\Theta$ dependence of the profile. From Eq. (2a), $R_{1 m}$ is comprised of low and high frequency contributions, the former proportional to $j\left(\omega_{I}\right)$, the latter proportional to $j\left(\omega_{D}\right)$, where $\omega_{D}$ is the electron spin transition frequency in the zfs limit. The relative contributions of the low and high frequency terms depend strongly on molecular geometry, the former as $3^{-1}\left[1+P_{2}(\cos \theta)\right] \cdot j\left(\omega_{I}\right)$ and the latter as $6^{-1}[2$ $\left.-P_{2}(\cos \Theta)\right] \cdot j\left(\omega_{D}\right)$. The two geometric functions are shown in Fig. 2. The low frequency part of $R_{1 m}$ [that proportional to $\left.j\left(\omega_{I}\right)\right]$ is largest for geometries in which the nuclear spin lies near the molecular $z$ axis $(\Theta=0)$. In equatorial positions $(\Theta=\pi / 2)$, this contribution drops to $1 / 4$ the axial value. The high frequency $R_{1 m}$ contribution varies in the opposite manner, its maximum occuring in equatorial positions and decreasing by a factor of $2 / 5$ in axial positions.

In addition to their opposite $\theta$ dependence, the low and high frequency $R_{1 m}$ contributions usually have very different magnitudes since, when $\omega_{D} \tau_{c}>1, j\left(\omega_{D}\right)<j\left(\omega_{I}\right)$. Thus in the zfs limit, it is normally the situation (as in Fig. 1) that the high frequency contribution is very small and that the $\Theta$ variation of Fig. 1 largely reflects the $\Theta$ dependence of the low frequency term. For this reason, the dispersion profiles in the zfs limit approach a constant form that is determined by the geometrical factor in the low

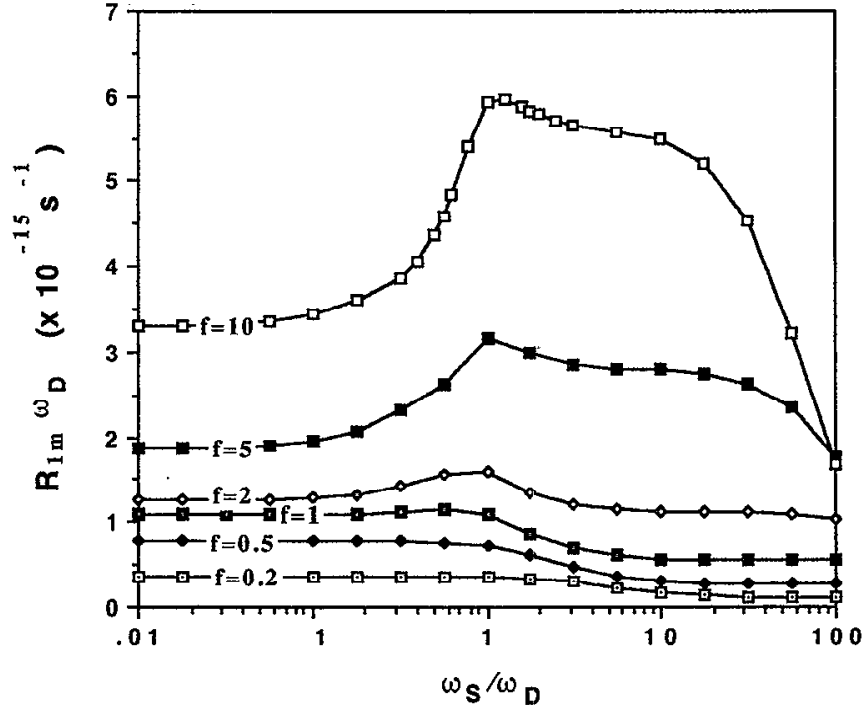

FIG. 3. Reduced field dispersion profiles for nuclear spins in an equatorial location $(\Theta=1.5)$ for the specified values of $\omega_{D} \tau_{c}$. Other parameters are the same as in Fig. 1.

frequency term of Eq. (2a); when $\omega_{D} \tau_{c} \gg 1$ (which is a common situation in the zfs limit), this form is independent of the correlation time.

This point is illustrated by the calculations of Fig. 3, which shows a family of dispersion profiles in which the position of the nuclear spin in the molecular axis system was fixed in an equatorial position $(\Theta=1.5)$ and $\omega_{D} \tau_{c}$ varied through the range $0.2-10$. When $\omega_{D} \tau_{c} \gg 1$, the reduced dispersion profiles approach a constant shape, similar to those in Fig. 1. The qualitative form of the profiles depends strongly on $\Theta$, but not on $\omega_{D} \tau_{c^{*}}$

\section{B. Physical origin of the feature centered near $\omega_{S} /$ $\omega_{D}=1$}

The qualitative form of the $(\Theta=1.5)$ curve in the neighborhood of $\omega_{S} / \omega_{D} \rightarrow 1$ in Fig. 1 is strikingly different from the dispersions of Zeeman-limit theory. For nuclei lying closer to the $z$ axis $(\Theta \cong 0)$, the form of the profile is qualitatively similar to that of Zeeman-limit theory, although in this case as well, the amplitudes and midpoint field values of the low-field features in the generalized theory and SBM theory differ considerably. In spite of the superficial similarity of the SBM and $\Theta \cong 0$ profiles, the low field featurcs in the Zeeman limit and generalized theories have fundamentally dissimilar physical origins. The former results from field dependence in the electronic transition frequency, which acts to suppress the spectral density terms $j\left(\omega_{S} \pm \omega_{I}\right)$ as $\omega_{S}$ rises. The latter type of feature arises from geometric effects associated with requantization of the electron spin. When $S$ is quantized along molecular axes, axial nuclear positions experience a different mean-squared dipolar field than do equatorial positions, and this difference is reflected in the magnitude of $R_{1 m}$. As $\omega_{S} / \omega_{D}$ rises through the cross-over region toward the Zeeman limit, the motion of $\bar{S}$ is requantized along the labo- 


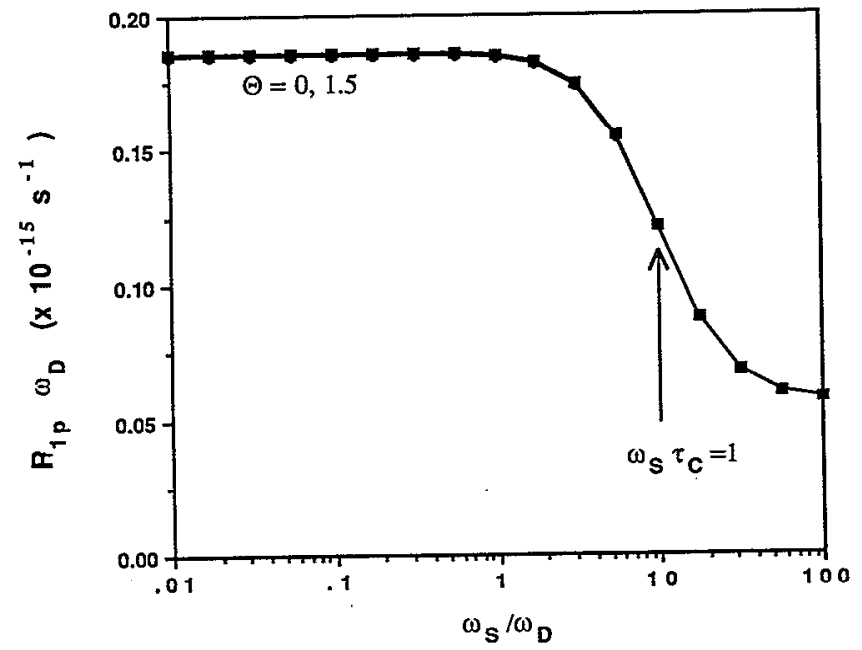

FIG. 4. Reduced field dispersion profiles calculated for $\Theta=0$ and $\Theta=1.5$ with $\omega_{D} \tau_{c}=0.1$. Other theoretical parameters are the same as for Fig. 1 . The two profiles very nearly superpose. Both show a Zeeman-type dispersive feature centered about $\omega_{S} \tau_{c}=1$.

ratory $z$ axis. In an isotropic liquid, the mean-squared magnetic dipole field at $I$ corresponds, in the Zeeman limit, to an average value over the surface of a sphere, thus eliminating the $\Theta$ dependence of $R_{1 m}$.

These two types of feature will be referred to as (1) Zeeman and (2) requantization. It is generally the case for $S=1$ that only one type of feature (not both) contributes to a given dispersion profile. This is physically evident from the fact that in the region where $\omega_{S}<\omega_{D}$, the electronic transition frequency $\omega_{m}$ is approximately field independent $\left(\omega_{m} \cong \omega_{D}\right)$, as is likewise the high frequency part of $R_{1 m}$, which is proportional to $j\left(\omega_{D}\right)$ rather than $j\left(\omega_{S}\right)$. In addition to its field independence $j\left(\omega_{D}\right)$ is typically small and remains so at very low field strengths. Thus the high frequency part of $R_{1 m}$ is negligible and the Zeemantype dispersion at $\omega_{S} \tau_{c}=1$ does not occur.

On the other hand, when the Zeeman-type feature at $\omega_{s} \tau_{c}=1$ occurs in the Zeeman-dominated region (this condition corresponds to $\omega_{D} \tau_{c}<1$ ), the high frequency part of $R_{1 m}$ is significant at low field and the type- 1 dispersion is present in the profile, while the type (2) dispersion (which is always located near $\omega_{S} / \omega_{D}=1$ ) disappears. This is illustrated by the profiles in Fig. 3, where the physical nature of the low-field feature changes from type (2) to type (1) when $\omega_{D} \tau_{c}$ drops below unity.

In summary, the low-field portion of the $S=1$ dispersion profile contains either a Zeeman-type feature or a requantization type feature, but not both. The Zeeman-type feature occurs when $\omega_{D} \tau_{c}<1$, the requantization-type feature when $\omega_{D} \tau_{c}>1$. To further illustrate this point, Fig. 4 shows dispersion profiles calculated for $\Theta=0$ and $\Theta=1.5$ with $\omega_{D} \tau_{c}=0.1$ and other parameters as in Fig. 1. The curves virtually (but not exactly) superpose, showing a Zeeman-type feature at $\omega_{S} \tau_{c}=1$, but no requantization feature at $\omega_{S} / \omega_{D}=1$. This situation is opposite to that in Fig. 1 , where $\omega_{D} \tau_{c}>1$ and where type 2 features occur.

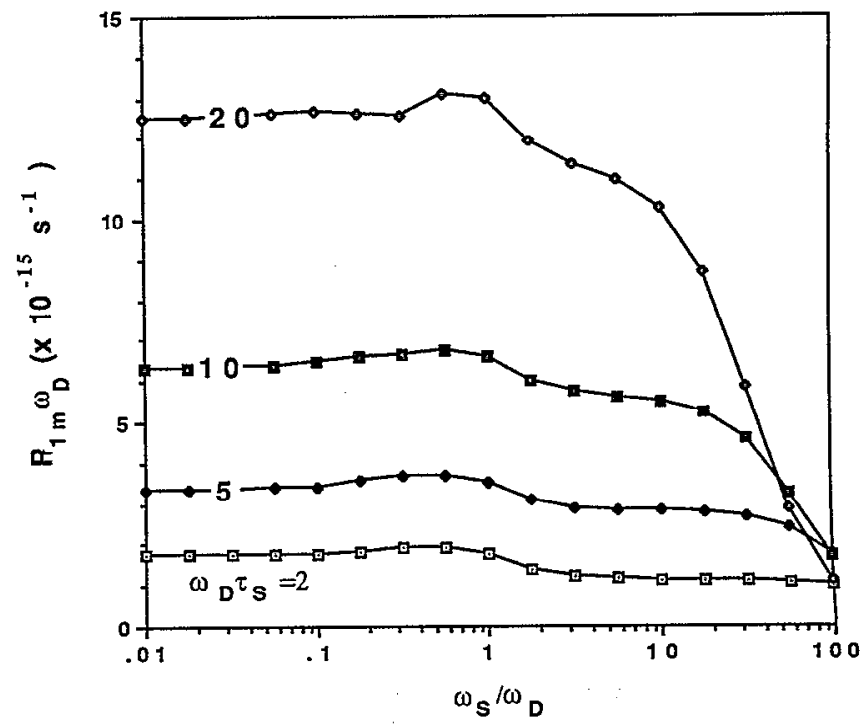

FIG. 5. Reduced field dispersion profiles of the NMR-PRE for $\Theta=0.955$. Curves were calculated for various fixed values of $\omega_{D} \tau_{c}$ in the range 2-20 using the program PARELAX (Ref. 6). Other theoretical parameters are given in the legend of Fig. 1.

These considerations lead to two distinct criteria for validity of Zeeman-limit (SBM) theory; namely, SBM theory is valid when (1) $\omega_{S} / \omega_{D} \gg 1$, or when (2) $\omega_{D} \tau_{c} \ll 1$. The first criterion is obvious physically. The second is less so; it states that when $\tau_{c} \ll \omega_{D}^{-1}$, effects of the zfs are unobservable in the dispersion profile and that Zeeman-limit theory is valid even at field strengths low enough that $\mathscr{H}_{\text {zfs }} \gg \mathscr{H}_{Z}$.

\section{The local $\boldsymbol{R}_{1 m}$ maximum in the intermediate regime}

In the intermediate regime between the limits, a curious local maximum in $\omega_{D} R_{1 m}$ occurs in the dispersion profiles for $\Theta=1.0$ and 1.5. This feature is apparent in Figs. 1 and 2. It is also present in Fig. 5, which shows a family of curves at specified values of $\omega_{D} \tau_{c}$ and a fixed value of $\Theta=0.955$. This $\Theta$ value corresponds to $P_{2}(\cos \theta)=0$. Since the average of $P_{2}(\cos \theta)$ over the surface of a sphere is zero, setting $\Theta=0.955$ in the zfs-limit calculation is equivalent to averaging the orientation of the $I-S$ vector (i.e., $\Theta$ ) in Eq. (2) over the surface of a sphere. At this $\Theta$ value, the mean-squared dipolar field at $I$ is the same in the zfs and Zeeman limits, and on this basis, a monotonic variation of $R_{1 m}$ between the limits would be expected. However, the computed profiles exhibit a local maximum in the intermediate region (Fig. 5). The physical origin of this maximum is evidently unrelated to alterations in the geometry of the magnetic dipole coupling.

Rather, it arises from the magnetic field dependence of the electron spin energy levels in the intermediate regime and, more specifically, from the crossing or "pinching" of energy levels that occurs when $\omega_{S} / \omega_{D}=1$ in nearly polar molecular orientations. This effect is illustrated in Fig. 6, which shows the $S=1$ energy levels as functions of magnetic field strength in different orientations of $B$ relative to $z$. ( $\theta$ is the polar angle of the molecular $z$ axis in the lab- 

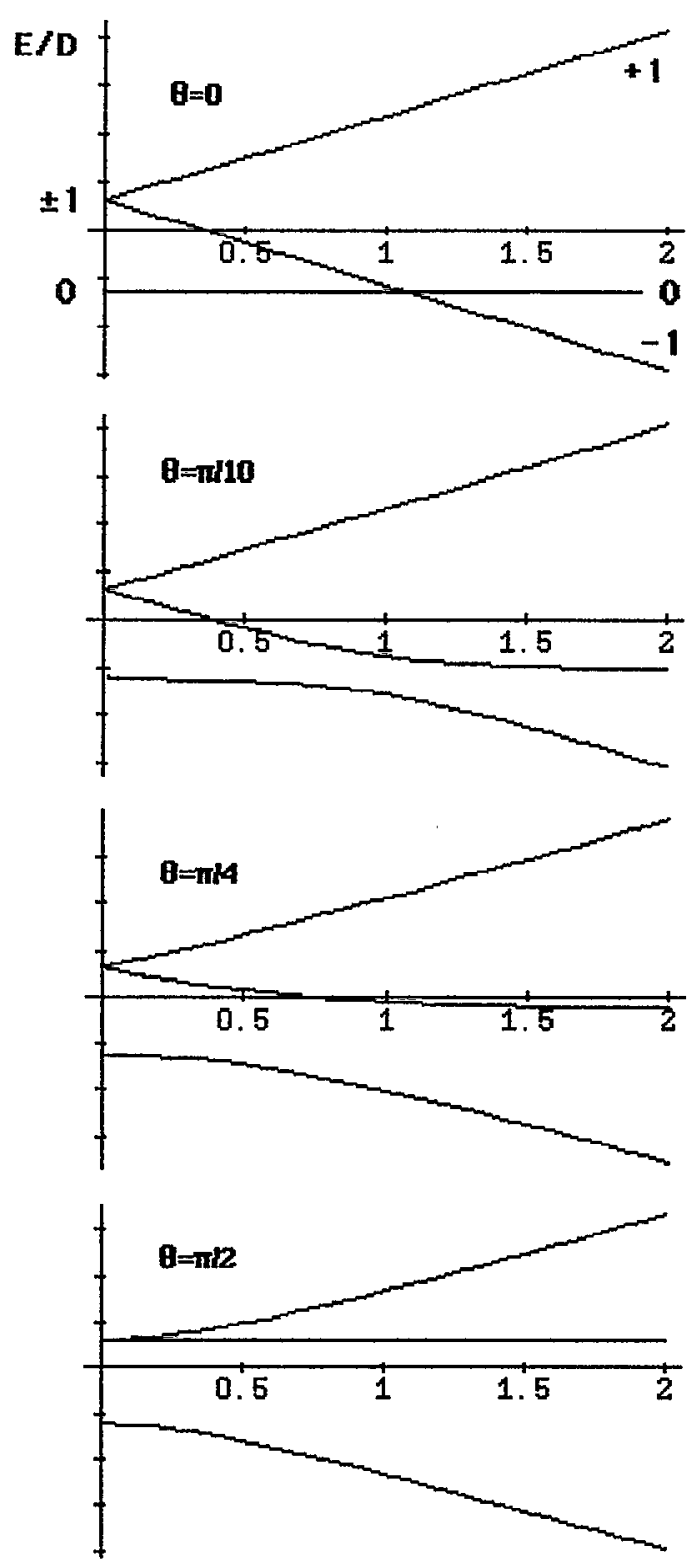

FIG. 6. Energy level diagrams for $S=1$ for various relative orientations of the molecular and laboratory $z$ axes. The horizontal coordinate is $\omega_{S} / \omega_{D}$, and the vertical coordinate is energy in units of $D$, where $D$ is the unixial $\mathrm{zfs}$ parameter.

oratory frame.) The high frequency part of $R_{1 m}$ results from single quantum transitions in the $S$ spin manifold, and crossings, or near crossings of the spin levcls increase the magnitude of this term. For $\theta=0$, there is a crossing of the $m_{s}=0$ and -1 levels near $\omega_{S} / \omega_{D}=1$. When $B$ and $z$ are not parallel, but $\theta$ is small (e.g., $\theta=\pi / 10$ ), the levels do not cross, but pinch together. These nearly parallel orientations produce a significant high frequency term in $R_{1 m}$ for molecular orientations that correspond to $\theta \cong 0$. This effect arises from molecules lying in a relatively small solid angle, but it clearly can be large enough to produce a significant local maximum in the field dispersion profile. To distinguish it from other physically distinct features, this type of feature will be referred to as type 3 .

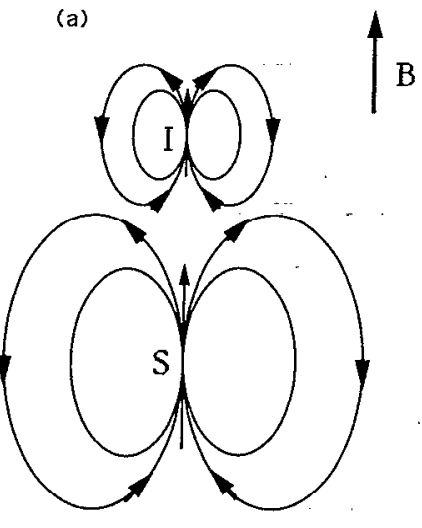

(b)
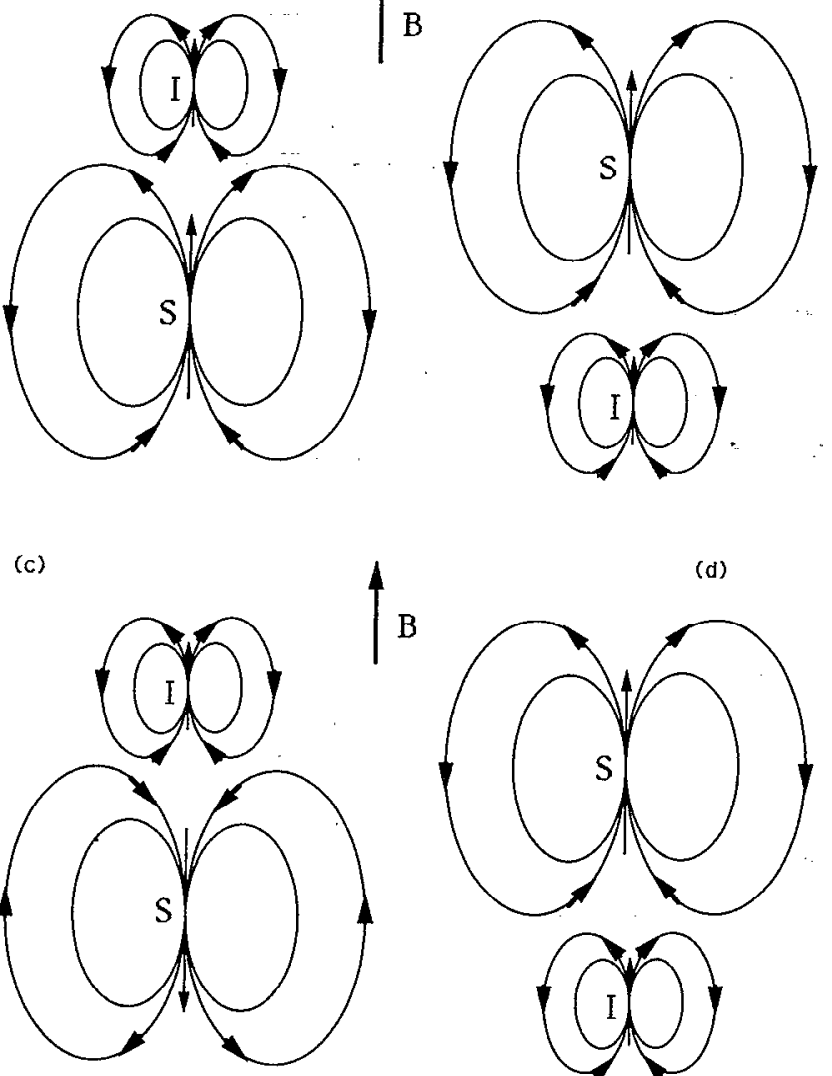

(d)

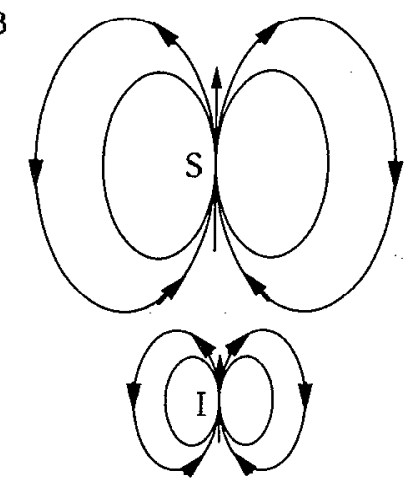

FIG. 7. The effect of requantization of the electron spin on the rotational symmetry of the nuclear-electron magnetic dipole interaction. $\overline{\mathbf{B}}$ is the static laboratory magnetic field. (a)-(d) show, schematically, the magnetic moments and the flux lines associated with the $I$ and $S$ spins. In the Zeeman limit [(a) and (b)], both $\overline{\mathbf{I}}$ and $\overline{\mathbf{S}}$ are quantized by the external field. A rotation (a) $\rightarrow$ (b) of the $I-S$ vector through an angle $\pi$ in the laboratory frame leaves the local magnetic field at $I$ due to $S$ unchanged. In the $\mathrm{zfs}$ limit (c) $\rightarrow$ (d), $\bar{S}$ is quantized along molecular axes. The same rotation causes an inversion of $\left\langle\bar{\mu}_{S}\right\rangle$ and of its associated local field at $I$.

\section{Magnetic field dependence of the reorientational correlation time}

It has been pointed out in earlier work ${ }^{4-6}$ that the definition of the reorientational correlation time differs in the zfs and Zeeman limits. In the Zeeman limit, $\tau_{R}=\tau_{R}^{(2)}$, which is the reorientational correlation time for a molecule-fixed second rank tensor. In the zfs limit, $\tau_{R}$ $=\tau_{R}^{(1)}$ for a first rank tensor. According to classical diffusion theory, ${ }^{19} \tau_{R}^{(1)}=3 \tau_{R}^{(2)}$. The change in definition of $\tau_{R}$ relates to an alteration of the symmetry of the magnetic dipole interaction under molecular rotation in the laboratory frame; the effect ultimately originates in the requantization of the electron spin. The relevant geometry is illustrated in Fig. 7. In the Zeeman limit [Figs. (7a) and (7b)], both the $S$ and $I$ spins are quantized along the laboratory $z$ axis by the external magnetic field. In this case, the magnetic dipole coupling energy (as well as the local field produced by $S$ ) are periodic under molecular rotations through angle $\pi$. In the zfs limit [Figs. (7c) and (7d)], the magnetic field associated with the $S$ spin is quantized along the molecular axes, while that of the $I$ spin 


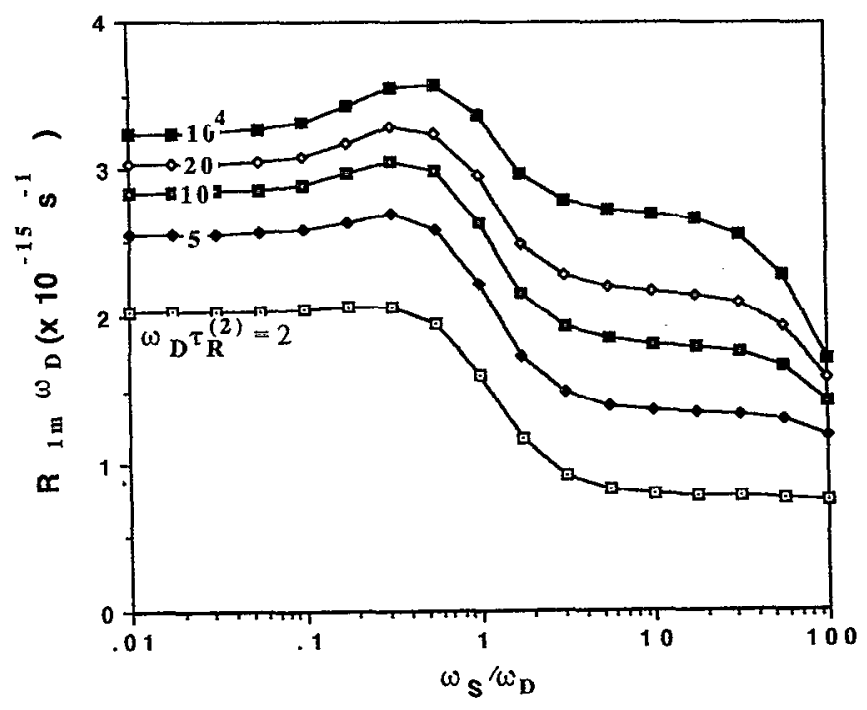

FIG. 8. Reduced field dispersion profiles of the NMR-PRE calculated for fixed $\Theta(\theta=0.955)$ and fixed $\tau_{c}\left(\omega_{D} \tau_{c}=5\right)$ with various assumed values of $\omega_{D} \tau_{R}$. The field dependence of $\tau_{R}$ was parametrized according to Eqs. (11) and (12) of the text. Other theoretical parameters are given in the legend of Fig. 1.

is quantized by the external field. Thus a rotation of the molecular axes through an angle $\pi$ inverts the direction of $\bar{\mu}_{S}$ as well as the sign of the local $S$ field at $I$. The local field of $S$ exhibits periodicity of $2 \pi$, the same as for a moleculefixed vector. Hence the change in definition of $\tau_{R}$ from $\tau_{R}^{(1)}$ to $\tau_{R}^{(2)}$ between the two physical limits.

In order to incorporate the redefinition of $\tau_{R}$ in the general theory, the following parametrized form of $\tau_{R}$ has been used:

$$
\left(\tau_{R}\right)^{-1}=\chi\left(\tau_{R}^{(1)}\right)^{-1}+(1-\chi)\left(\tau_{R}^{(2)}\right)^{-1}
$$

$\chi$ is a parameter which tracks the progress from the Zeeman limit $(\chi=0)$ to the $\mathrm{zfs}$ limit $(\chi=1)$ in a suitable manner. Setting

$$
\chi \equiv \sin ^{2}\left(\frac{\pi}{2} \cdot \frac{\omega_{S}}{\omega_{S}+\omega_{D}}\right)
$$

ensures the required behavior at the limits, as well as that $\chi=0.5$ when $\omega_{S}=\omega_{D}$.

When $\tau_{R}$ contributes significantly to $\tau_{c}$, the field dependence of $\tau_{R}$ can produce an important qualitative feature in the dispersion profile, namely a drop in $\omega_{D} R_{1 m}$ which occurs when $\omega_{S} / \omega_{D} \cong 1$. This effect is illustrated in Fig. 8, which shows curves calculated for $\omega_{D} \tau_{R}=2,5,10$, 20 , and $10^{4}$ using $1 / \omega_{D} \tau_{C}=1 / \omega_{D} \tau_{S}+1 / \omega_{D} \tau_{R}$ in conjunction with the parametrized form of $\tau_{R}$. $\Theta$ was set equal to 0.955 to eliminate the effects due to changes in the meansquare dipolar coupling energy, and $\omega_{D} \tau_{S}$ was set equal to 5. Significant effects of $\tau_{R}$ on the shape of the profile occur when $\tau_{R} / \tau_{S} \lesssim 1$. Thus the curve for $\omega_{D} \tau_{R}=10^{4}$ is very similar to that for $\Theta=1.0$ of Fig. 1, where $\tau_{c}$ was taken to be $5 / \omega_{D}$ and field independent. As $\tau_{R} / \tau_{S}$ decreases, the drop in $R_{1 m}$ becomes more pronounced, both relatively and absolutely, approaching a factor of $1 / 3$ when $\tau_{R} \ll \tau_{S}$. Fea-

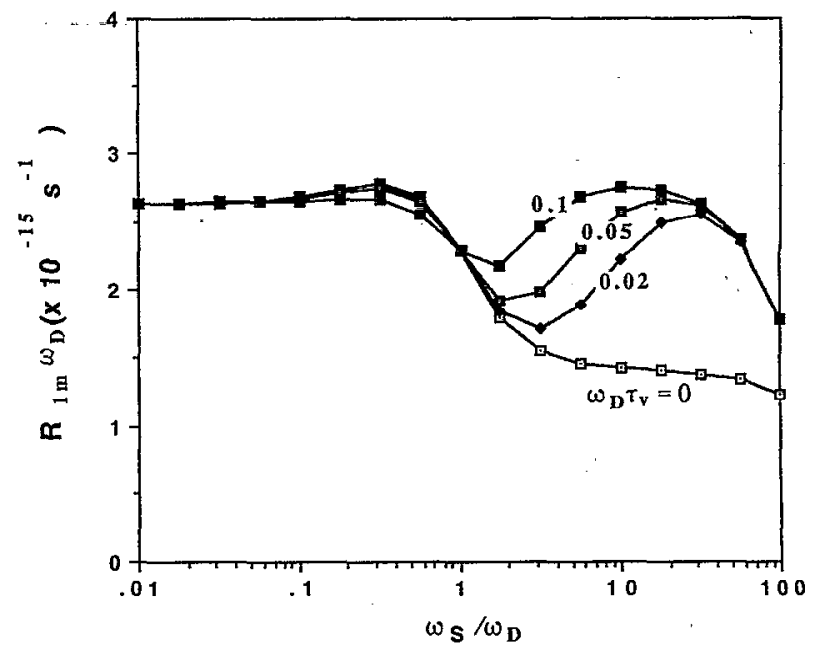

FIG. 9. Reduced field dispersion profiles of the NMR-PRE calculated for fixed $\Theta(\Theta=0.955)$, fixed $\tau_{S}\left(\omega_{D} \tau_{S}=5\right)$, fixed $\tau_{R}\left(\omega_{D} \tau_{R}=5\right)$, and various values of $\omega_{D} \tau_{v}$ in the range $0.0-0.1$. Other theoretical parameters are given in the legend of Fig. 1.

tures of this type, which arise from a dependence of the definition of $\tau_{R}$ on magnetic field strength, will be referred to as type- 4 features.

\section{E. Field dependence of $\tau_{S}$}

Bloembergen and Morgan ${ }^{3}$ showed that $\tau_{S}$ becomes field dependent at high field strengths as given by

$$
\frac{1}{\tau_{S}}=\frac{1}{\tau_{S}^{(0)}}\left(\frac{0.2}{1+\omega_{S}^{2} \tau_{v}^{2}}+\frac{0.8}{1+4 \omega_{S}^{2} \tau_{v}^{2}}\right)
$$

where $\tau_{S}^{(0)}$ is the low field limit of $\tau_{S}$. This form is valid for the Zeeman limit when relaxation is produced by stochastic time dependence, with a correlation time $\tau_{v}$, in the quadratic $\mathrm{zfs}$ interaction. Generalizations of the theory that account for zero field splittings in the static electron spin Hamiltonian have been developed. ${ }^{22-26}$ As a general phenomenon, the field dependence of the spin energy levels produces field dependence in $\tau_{S}$ at sufficiently high field strengths (typically $>1-2 \mathrm{~T}$ when the static zfs is small), causing $\tau_{S}$ to increase in the high field limit as $B^{2}$.

The general effect of this field dependence on the shape of the reduced dispersion profile is illustrated in Fig. 9. When $\tau_{S}$ enters its field-dependent regime $\left(\omega_{S} \tau_{v}>1\right), \tau_{S}$ and $R_{1 m}$ first increase as $B_{0}^{2}$ and then decrease as $B_{0}^{-4}$, passing through a local maximum near $\omega_{S} \tau_{S}=1$. This local maximum is a well-known characteristic of the Zeemanlimit (SBM) theory; by extension of the terminology used above, it is a type- 5 feature. It should be noted, although we do not pursue the subject in detail here, that Eq. (13) is valid only for the Zeeman limit. When the static zfs is large enough that the onset of the field-dependent regime for $\tau_{S}$ (i.e., $\omega_{S} \tau_{v} \cong 1$ ) occurs outside the Zeeman limit, the field dependence of $\tau_{S}$ is much milder than that predicted by Eq. (13), the $R_{1 m}$ maximum being suppressed. The influence of the $\mathrm{zfs}$ in suppressing the field dependence of $\tau_{S}$ has been noted experimentally in tris-acetylacetonato- 
metal complexes, ${ }^{5,25}$ where the static $\mathrm{zfs}$ is moderately large $|D|=2-5 \mathrm{~cm}^{-1}$. In these cases $R_{1 m}$ is approximately field independent at quite high field strengths (several Tesla) in apparent contradiction of Eq. (13).

In principal, an additional source of field dependence in $\tau_{S}$ occurs at the boundary of the Zeeman and zfs limits, since the relaxation of the $S$ spin is defined with respect to the axes of its precessional motion, and these axes change when the $S$ spin undergoes requantization. In practice, however, this kind of effect is likely to be very small, in most cases, probably insignificant. For spins $S \geqslant 1, \tau_{S}$ results physically from modulation of the zfs tensor by vibrational, collisional, and reorientational motions. Many degrees of freedom contribute in a way that is complex and difficult to analyze quantitatively. However, it seems likely that in most cases relaxation will be approximately isotropic with respect to molecular axes. When this is true, only a single relaxation parameter, field dependent in the Zeeman limit as given by Eq. (13), but field independent in the intermediate regime $\left(\omega_{S} / \omega_{D} \cong 1\right)$, need be used.

\section{THEORETICAL INTERPRETATION OF THE PRE OF $\mathrm{Ni}\left(\mathrm{H}_{2} \mathrm{O}\right)_{6}^{2+}$}

The ${ }^{1} H$ and ${ }^{2} H$ NMR-PRE due to the aqueous Ni (II) ion have been studied ${ }^{11,13-17}$ as functions of temperature, magnetic field strength, and $\mathrm{pH}$ over a wide range of magnetic field strengths $(0.047-11.7 \mathrm{~T})$. The inner sphere proton relaxation rate $R_{1 m}$ has been particularly well characterized at 51 and $71^{\circ} \mathrm{C}$, where chemical exchange is relatively rapid and its effects negligible. At these temperatures, the dispersion profile exhibits a local maximum near $0.6 \mathrm{~T}$ and a local minimum near $4 \mathrm{~T}$ as described above. These profiles have been reinterpreted theoretically in terms of intramolecular dipolar and scalar relaxation contributions. In previous studies, ${ }^{13,15,17}$ the effects of scalar relaxation and outer sphere dipolar relaxation were estimated and found to be negligible. We reassessed the scalar contribution, since in the $\mathrm{zfs}$ limit, $R_{\mathrm{I} m, \mathrm{sc}}$ responds to low frequency portions $j\left(\omega_{I}\right)$ of the spectral density function and can be relatively much more efficient than in the Zeeman limit. ${ }^{4}$ Calculations of $R_{1 m, s c}$ were carried out us: ing a scalar coupling constant $(A / 2 \pi)=0.13 \mathrm{MHz}$ based on contact shift measurements ${ }^{15}$ and a $\tau_{S}$ value of $\sim 10-20$ ps (see below). $R_{1 m, \text { sc }}$ was found, as in previous studies, to be very small ( $<1 \%$ of $R_{1 m \text {,dip }}$ ), even in the zfs limit, thus justifying neglect of this term.

The experimental dispersion profiles were simulated in terms of the inner sphere dipolar contribution using the generalized theory of Ref. 6. As in previous studies, ${ }^{11,17}$ it was assumed that the electronic spin of $\mathrm{Ni}\left(\mathrm{H}_{2} \mathrm{O}\right)_{6}^{2+}$ experiences a static $\mathrm{zfs}$ splitting. Since the static zfs vanishes in cubic site symmetry, this implies that the hydration shell has distorted octahedral structures corresponding to local energy minima. The existence of a nonzero zfs also implies the lifetime of these structures be longer than the reciprocal zfs transition frequency $\tau_{a}>\omega_{D}^{-1}$. As an example, for $D=1 \mathrm{~cm}^{-1}$, this corresponds to $\tau_{a}>5 \mathrm{ps}$. This kind of condition should be easily met if the distorted structure lies in an energy minimum of a few hundred calories. The

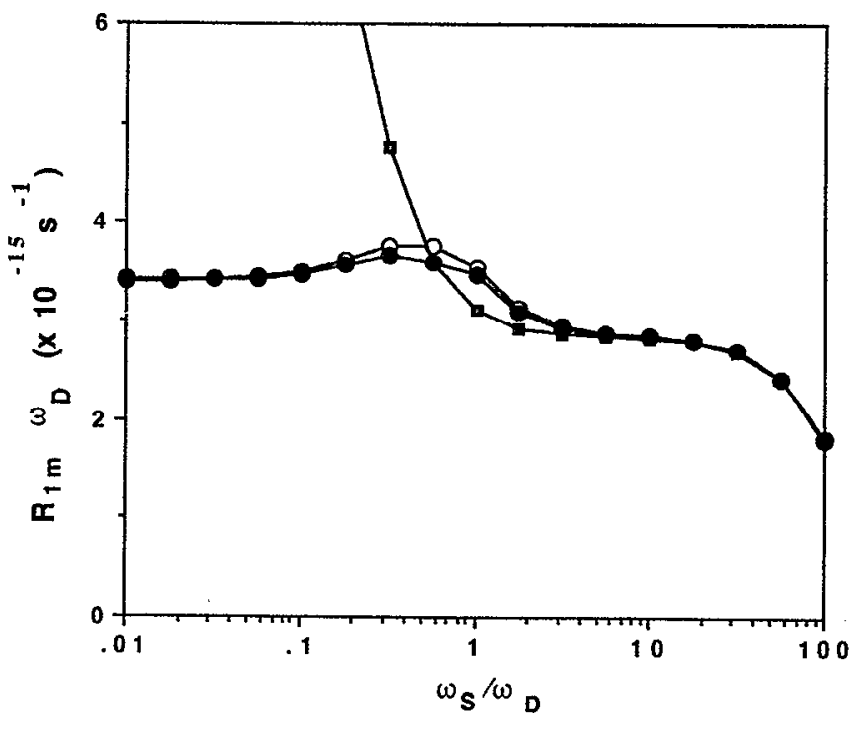

FIG. 10. The effect of spatial averaging on the reduced field dispersion profiles of the NMR-PRE. Open circles are a profile in which $R_{\mathrm{t} m}$ values were calculated using $\Theta=0.955$, which is the average value of $\Theta$ on the hemisphere corresponding to $0 \leqslant \theta \leqslant \pi / 2$. Closed circles give the profile in which each $R_{1 m}$ value was averaged over the space of the $I-S$ vector $0 \leqslant \Theta \leqslant \pi$. The dipolar correlation time was $\omega_{D} \tau_{c}=5$ (field independent); other theoretical parameters are given in the legend of Fig. 1.

hydration energy of $\mathrm{Ni}(\mathrm{II})$ is about $40 \mathrm{kcal} \mathrm{mol}^{-1}$ per water molecule, ${ }^{27}$ and it seems very likely that energy minima of at least that magnitude exist. It is of course possible that more than one minimum exists, and if so, the $\mathrm{zfs}$ parameter obtained from NMR-PRE data corresponds to a structural average.

To incorporate effects of the $\mathrm{zfs}$ into the calculation, the molecular geometry must be defined in terms of an appropriate $\Theta$ value. The coordination sphere of aqueous $\mathrm{Ni}$ (II) contains six water molecules in a distorted octahedral configuration. ${ }^{28,29}$ It seems reasonable to assume that the 12 water protons samplc, approximately, an averaged environment over the surface of the coordination sphere. In this situation, the theoretical $R_{1 m}$ values must be averaged with respect to the space of $\Theta$. This procedure is computationally rather time consuming, however, and an alternate procedure, in which the spatially averaged value $\Theta_{\mathrm{av}}=0.955$ of $\Theta$ in the interval $0 \leqslant \theta \leqslant \pi / 2$ was used instead. The difference in these averaging procedures was explored in trial calculations (Fig. 10) and found to be small.

Theoretical fits to the experimental profiles at 51 and $71{ }^{\circ} \mathrm{C}$ are shown in Figs. 11 and 12, and the associated parameter sets are given in Table I. Table I also gives, for comparison, the parameters of the unconstrainted fits of Ref. 17. In the fit using PARELAX (Figs. 11 and 12), $r$ was fixed at $0.2657 \mathrm{~nm}$, a value suggested by $\mathrm{x}$-ray and neutron diffraction studies, ${ }^{13}$ and $\Theta$ was taken to be $\Theta_{a v}$ $=0.955$. The zfs parameter required by the fit was about $2.15 \mathrm{~cm}^{-1}\left(\omega_{D}=0.405 \times 10^{12} \mathrm{rad} \mathrm{s}^{-1}\right)$. Thesc values of $r$, $\Theta$, and $D$ provided a satisfactory fit to both the 51 and $71^{\circ} \mathrm{C}$ data. The needed temperature dependence resided 


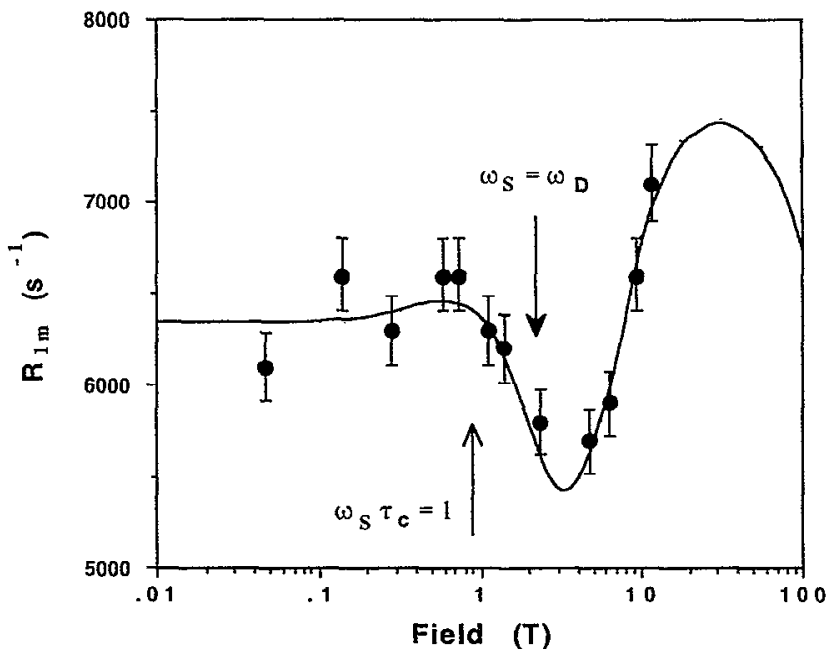

FIG. 11. A fil of the theory to proton $R_{1 m}$ data for $\mathrm{Ni}\left(\mathrm{H}_{2} \mathrm{O}\right)_{6}^{2}$ at $51^{\circ} \mathrm{C}$. Data are taken from Refs. 16 and 17. Uncertainties shown correspond to $\pm 3 \%$ of data (Ref. 17). The solid line is a theoretical curve calculated using the theory of Ref. 6, assuming $D=2.151 \mathrm{~cm}^{-1}\left(\omega_{D}=0.405 \times 10^{12}\right.$ $\left.\mathrm{rad} \mathrm{s}{ }^{-1}\right), \Theta=0.955 \mathrm{rad}, r_{I S}=0.2657 \mathrm{~nm}, \tau_{v}=1.14 \mathrm{ps}, \tau_{R}^{(2)}=13.6 \mathrm{ps}$, and $\tau_{S}^{(0)}=11.4 \mathrm{ps}$. Arrows indicate the cross-over point $\omega_{S}=\omega_{D}$ between the Zeeman and zfs limits, and the midfield point $\omega_{S} \tau_{c}=1$ of the type 1 (Zeeman) feature.

entirely in $\tau_{R}, \tau_{S}^{(0)}$, and $\tau_{v}$. The parameter $\tau_{R}^{(2)}$ shortened with increasing temperature by about $25 \%$, which is in accord with the predicted variation of Debye's theory, ${ }^{21}$ in which $\tau_{R}^{(2)} \propto$ (viscosity/temperature). $\tau_{v}$ decreased slightly and $\tau_{S}^{(0)}$ increased slightly with temperature in a physically reasonable manner.

It is difficult to compare in detail the theory (S.M.) of Ref. 17, which is based on the stochastic Liouville approach, with that used here, but the application of these theories to the aqueous $\mathrm{Ni}$ (II) data seems to lead to rather different results. The problem is not so much one of achieving low rms error in the fitting procedure; all three theories SBM, S.M., and PARELAX produce functional forms which fit the data with acceptable rms error. However, only PARELAX predicts qualitatively the observed low field rise in $R_{1 m}$, which is clearly a type-3 feature. The fitting parameters from PARELAX are also more reasonable than those of the SBM and S.M. approaches. In the latter, the required $I S$ distance is quite small, probably

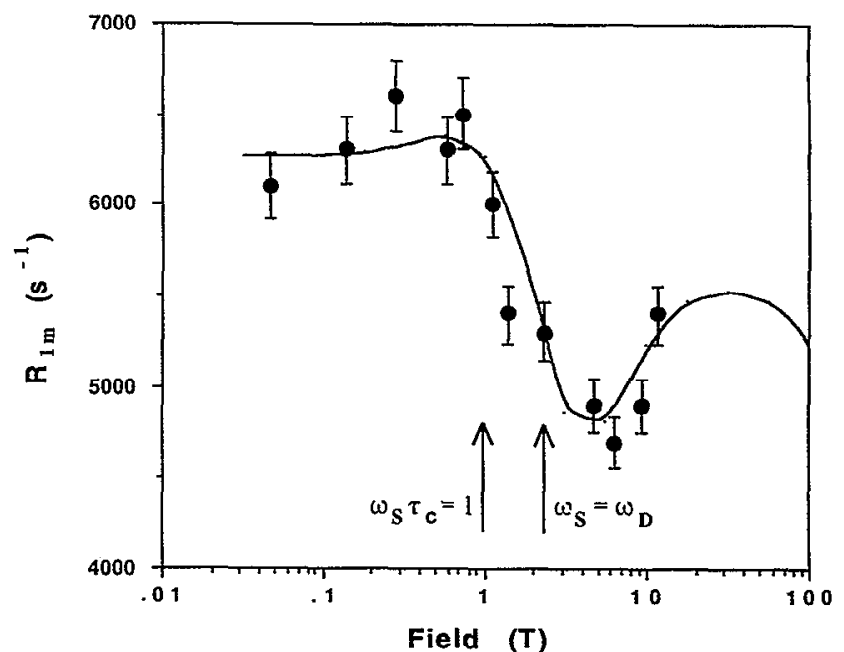

FIG. 12. A fit of the theory to proton $R_{1 m}$ data (Refs. 16 and 17) for $\mathrm{Ni}\left(\mathrm{H}_{2} \mathrm{O}\right)_{6}^{2+}$ at $71^{\circ} \mathrm{C}$. The solid line is a theoretical curve calculated using the theory of Ref. 6 , assuming $D=2.151 \mathrm{~cm}^{-1}, \Theta=0.955 \mathrm{rad}, r_{I S}$ $=0.2657 \mathrm{~nm}, \tau_{v}=1.00 \mathrm{ps}, \tau_{R}^{(2)}=10.0 \mathrm{ps}$, and $\tau_{S}^{(0)}=12.4 \mathrm{ps}$.

unphysically so, and both this distance and $\omega_{D}$ exhibit substantial unexpected temperature dependence. In addition, the temperature dependence of $\tau_{v}$, which was found to lengthen with increasing temperature, varies in a direction that is clearly unphysical. These problems did not occur in the PARELAX fit; the data at both 51 and $71{ }^{\circ} \mathrm{C}$ were fit satisfactorily using constant, physically reasonable values of $r$ and $\omega_{D}$, and the required temperature dependencies of $\tau_{R}^{(2)}, \tau_{S}^{(0)}$, and $\tau_{v}$ were as expected. Another more basic difference between the theories rclates to the underlying physical interpretation (see below).

The derived value of $\omega_{D}$, which corresponds to $D=2.151 \mathrm{~cm}^{-1}$, is within the expected range for hexaquo$\mathrm{Ni}(\mathrm{II})$, although specific quantitative values cannot be extracted from the solid state literature since the magnitude of $D$ appears to be a sensitive function of the specific hydration environment of the ion..$^{30}$ For example, for crystalline $\mathrm{Ni}$ (II) $\mathrm{SO}_{4} \cdot 6 \mathrm{H}_{2} \mathrm{O}, D=4.85 \mathrm{~cm}^{-1}$ (Ref. 31), while for $\mathrm{NiSiF}_{6} \cdot\left(\mathrm{H}_{2} \mathrm{O}\right)_{6},|D|=0.5 \mathrm{~cm}^{-1}$ (Ref. 32). Theoretical calculations ${ }^{33}$ have indicated that $D$ depends strongly on small displacements of the water molccules and suggest

TABLE I. Fitting parameters for the aqueous Ni(II) data obtained using Zeeman-limit (SBM) theory (Ref. 17), the unconstrained slow motion pseudorotation model (S.M.) of Ref. 17, and calculations of this work (PARELAX) based on the theory of Ref. 6. $\tau_{s}^{(0)}$ was not specified (n.s.) in Ref. 17; values shown were recomputed from SBM theory and agree with the low-field NMR-PRE data. $r$ was not permitted to vary in the calculations using PARELAX.

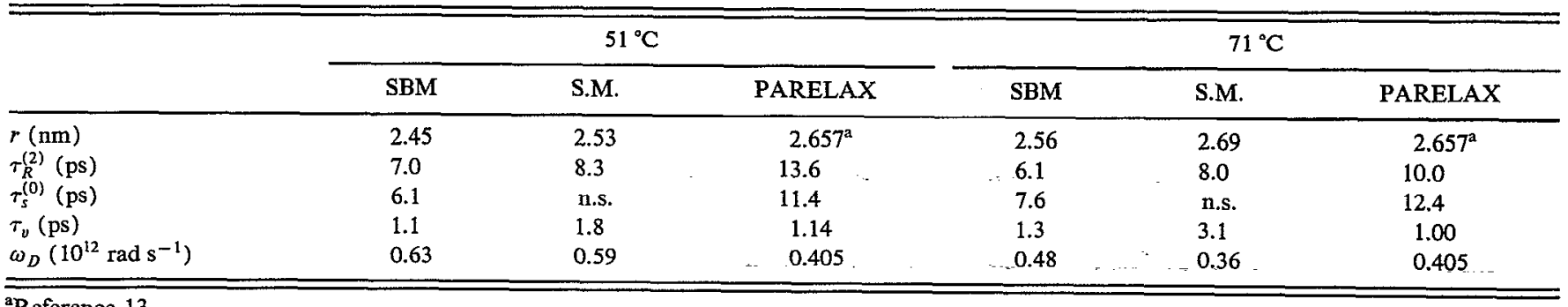

${ }^{\mathrm{a}}$ Reference 13 . 
that typical values of $D$ lie in the range one to several $\mathrm{cm}^{-1}$, consistent with the findings reported here as well as in previous NMR-PRE studies. ${ }^{11,17}$

\section{Physical interpretation of the field dispersion profile}

PARELAX successfully simulated the experimental profiles with respect to the qualitative features, namely, the gradual rise in $R_{1 m}$ below $1 \mathrm{~T}$, which is a type- 3 feature (pinching of the energy levels); the subsequent $R_{1 m}$ drop between 1 and $4 \mathrm{~T}$, which is primarily a type-4 feature (field dependence in $\tau_{R}$ ); and the $R_{1 m}$ rise above $5 \mathrm{~T}$, which is a type- 5 feature (Zeeman-type field dependence in $\left.\tau_{S}\right)$. The observed drop in $R_{1 m}$ at $\sim 1 \mathrm{~T}$ basically marks the cross-over point between the zfs and Zeeman regimes ( $\omega_{S}$ $\left.=\omega_{D}\right)$. However, a clear distinction between the requantization features (types 2, 3, and 4) on the one hand, and the Zeeman feature (type 1) on the other, is well defined only when the cross-over point $\left(\omega_{S}=\omega_{D}\right)$ is substantially to high field of the midfield point of the type-1 dispersion $\left(\omega_{s} \tau_{c}=1\right)$. In the aqueous $\mathrm{Ni}$ (II) system, these points are not widely separated (arrows in Figs. 11 and 12). Thus the features of the experimental profiles are of mixed type, although the zfs character predominates.

${ }^{1}$ I. Solomon, Phys. Rev. 99, 559 (1955).

${ }^{2}$ N. Bloembergen, J. Chem. Phys. 27, 572 (1957); 27, 595 (1957).

${ }^{3}$ N. Bloembergen and L. O. Morgan, J. Chem. Phys. 34, 842 (1961).

${ }^{4}$ R. R. Sharp, J. Chem. Phys. 93, 6921 (1990).

${ }^{5}$ T. Bayburt and R. R. Sharp, J. Chem. Phys. 92, 5892 (1990).

${ }^{6}$ R. R. Sharp, J. Magn. Reson. 100, 491 (1992).
${ }^{7}$ N. Benetis, J. Kowaleski, L. Nordenskiold, H. Wennerstrom, and P.-O. Westlund, Mol. Phys. 48, 329 (1983).

${ }^{8}$ N. Benetis and J. Kowaleski, J. Magn. Reson. 65, 13 (1985).

${ }^{9} \mathrm{~N}$. Benetis, J. Kowalewski, L. Nordenskiold, H. Wennerstrom, and P.-O. Westlund, Mol. Phys. 50, 515 (1983).

${ }^{10}$ U. Lind̈ner, Ann. Phys. (Leipzig) 16, 319 (1965).

${ }^{11}$ H. L. Friedman, M. Holz, and H. G. Hertz, J. Chem. Phys. 70, 3369 (1979).

${ }^{12}$ L. O Morgan and A. W. Nolle, J. Chem. Phys. 31, 365 (1959).

${ }^{13}$ D. B. Bechtold, G. Liu, H. W. Dodgen, and J. P. Hunt, J. Phys. Chem. 82, 333 (1978).

${ }^{14}$ J. W. Neely and R. E. Connick, J. Am. Chem. Soc. 94, 3420 (1972).

${ }^{15}$ J. Granot, A. M. Achlama, and D. Fiat, J. Chem. Phys. 61, 3043 (1974).

${ }^{16}$ H. G. Hertz and M. Holz, J. Magn. Reson. 63, 64 (1985).

${ }^{17}$ J. Kowalewski, T. Larsson, and P.-O. Westlund, J. Magn. Reson. 74, 56 (1987).

${ }^{18}$ P.-O. Westlund, N. Benetis, and H. Wennerstrom, Mol. Phys. 61, 177 (1987).

${ }^{19}$ W. T. Huntress, Adv. Nucl. Magn. Reson. Spectrosc. 4, 2 (1970).

${ }^{20}$ Z. Luz and S. Meiboom, J. Chem. Phys. 40, 2686 (1965).

${ }^{21}$ A. Abragam, The Principles of Nuclear Magnetism (Oxford University, Oxford, 1961), Chap. 8.

${ }^{22}$ A. D. McLachlan, Proc. R. Soc. London Ser. A 280, $271^{\circ}$ (1964).

${ }^{23}$ D. T. Pegg and D. M. Doddrell, Aust. J. Chem. 29, 1869 (1976).

${ }^{24}$ D. T. Pegg and D. M. Doddrell, Aust. J. Chem. 31, 475 (1978).

${ }^{25}$ D. T. Pegg, D. M. Doddrell, M. R. Bendall, and A. K. Gregson, Aust. J. Chem. 291885 (1976).

${ }^{26}$ J. H. Freed, G. V. Bruno, and C. Polnaszek, J. Chem. Phys. 55, 5270 (1971).

${ }^{27}$ M. Rosi and C. W. Bauschlicher, Jr., J. Chem. Phys. 90, 7264 (1989).

${ }^{28}$ B. H. O'Connor and D. H. Dale, Acta Crystallogr. 21, 705 (1966).

${ }^{29}$ H. L. Friedman and L. Lewis, J. Solut. Chem. 5, 445 (1976).

${ }^{30}$ A. Bencini and D. Gatteschi, Trans. Metal Chem. 7, 1 (1982).

${ }^{31}$ W. M. Pontuschka, A. Pinccini, J. A. Quadros, and S. Isotani, Phys. Lett. A 44, 57 (1973).

${ }^{32}$ R. S. Rubins, J. D. Clark, and S. K. Jani, J. Chem. Phys. 67, 893 (1977).

${ }^{33}$ C. Ribbing, M. Odelius, A. Laaksonen, J. Kowalewski, and B. Roos, Int. J. Quantum Chem, Quantum Chem. Symp. 24, 295 (1990). 